\title{
Control social en el cumplimiento normativo del sistema INFOBRAS en la Entidad Prestadora de Servicios de Saneamiento Tacna S.A.
}

\author{
Social control in the regulatory compliance of the INFOBRAS system in the entity \\ provider of sanitation services Tacna S.A.
}

\author{
Dennys Geovanni Calderón Paniagua \\ Universidad Cesar Vallejo \\ Lima, Perú \\ dennyscalderon13@hotmail.com \\ https://orcid.org/0000-0002-6569-0634
}

\author{
Juan Francisco Pacompia Toza \\ Universidad José Carlos Mariátegui \\ Tacna, Perú \\ juanpacompia2@hotmail.com \\ https://orcid.org/0000-0003-1993-5407
}

[Presentado: 30/01/2021, Aceptado: 16/02/2021]

\section{RESUMEN}

El objetivo de esta investigación fue la evaluación, mediante el control social, del cumplimiento normativo del sistema INFObras en la Entidad Prestadora de Servicios de Saneamiento Tacna S.A (EPS Tacna S.A.). La investigación fue de tipo básica, con un diseño de nivel descriptivo - explicativo, ya que buscó la generación de conocimiento evaluando el nivel de cumplimiento normativo del registro de obras públicas en el sistema INFObras en la EPS Tacna S.A. durante el periodo 2013-2020, fortaleciendo el control y la transparencia de las obras públicas con el fin de favorecer el control social por parte de la ciudadanía. La población estuvo constituida por las 86 obras públicas registradas en el sistema INFObras y los 17 proyectos de inversión registrados en el sistema de seguimiento de la ejecución presupuestal del Ministerio de Economía y Finanzas (MEF). Se concluyó que la aplicación del control social en la evaluación de la información del sistema INFObras de la Contraloría General de la República del Perú (CGR) y del sistema de ejecución presupuestal del MEF permitió evidenciar un alto nivel de incumplimiento normativo en el registro de obras públicas del sistema INFObras, por parte de la EPS Tacna S.A.; limitando el derecho a la participación ciudadana de la población de la región Tacna, es decir, tener la posibilidad de conocer si las obras en su región se estén desarrollando dentro de los plazos establecidos, con la calidad requerida y cumpliendo su finalidad pública.

Palabras clave: Cumplimiento, INFobras, normatividad, obras, proyectos.

\begin{abstract}
The objective of this research was the evaluation, through social control, of the regulatory compliance of the INFObras system at the Entidad Prestadora de Servicios de Saneamiento Tacna S.A. (EPS Tacna S.A.). The research was of a basic type, with a descriptive explanatory level design, since it sought to generate knowledge by evaluating the level of regulatory compliance with the registration of public works in the INFObras system in EPS Tacna S.A. during the period 2013 - 2020, strengthening the control and transparency of public works in order to favor social control by citizens. The population consisted of the 86 public works registered in the INFObras system and the 17 investment projects registered in the budget execution monitoring system of the Ministry of Economy and Finance (MEF). It was concluded that the application of social control in the evaluation of information from the INFObras system of the Comptroller General of the Republic of Peru (CGR) and the budget execution system of the MEF revealed a high level of noncompliance in the registration of public works in the INFObras system by EPS Tacna S.A., limiting the right to citizen participation of the population of the Tacna region, that is, to have the possibility of knowing if the works in their region are being developed within the established timeframe, with the required quality and fulfilling their public purpose.
\end{abstract}

Keywords: Compliance, INFobras, regulations, works, projects.

Este es un artículo de acceso abierto, distribuido bajo los términos de la Licencia Creative Commons Atribución 4.0 Internacional. (CC BY 4.0). https://creativecommons.org/licenses/by/4.0/deed.es 


\section{INTRODUCCIÓN}

Lograr que las entidades públicas en todos los niveles del Estado lleguen a ser eficientes y transparentes en la rendición de cuentas es una de las grandes metas aún inconclusas para generar valor público, no solamente en el Perú sino a nivel internacional, tal es así que esta problemática se encuentra priorizada dentro de los objetivos de desarrollo sostenible al año 2030 (Organización de las Naciones Unidades, 2015), exactamente en el objetivo 16: paz, justicia e instituciones públicas.

Actualmente, somos testigos de diversos actos de corrupción, que vienen haciéndose visibles a nivel nacional e internacional, por porte de grandes empresas, grupos de poder y organizaciones criminales organizadas que no permiten generar confianza en la ciudadanía, siendo el sector estatal uno de los más desprestigiados. Tal situación genera un descontrol y desorden al no tener la seguridad del destino final de los impuestos que la ciudadanía realiza, sobre todo al no verlos materializados en el contexto social, por ejemplo, en la ejecución de obras.

Más aún con la aparición de la COVID-19, que ha cambiado radicalmente nuestro forma de vivir, priorizando en el sector público (en su política de lucha contra la corrupción) la inserción de mecanismos virtuales para el desarrollo de sus funciones; impulsando de forma concordarte la implementación del gobierno electrónico, uno de los tres ejes transversales de la modernización de la gestión pública (MGP) en nuestro país. Esto sobre todo porque últimamente las entidades públicas han perdido la credibilidad debido a problemas de corrupción, soborno, entre otros males endémicos que se han hecho visibles.

En ese sentido, la participación ciudadana debe ser un bastión en la lucha contra la corrupción, fortaleciéndose desde todos los niveles del Estado. Debe existir el compromiso, por parte del ciudadano, de la protección y defensa de los intereses comunes para mitigar los problemas públicos. Sin embargo, lastimosamente, en esta última década no se ve un real compromiso del ciudadano por insertarse en la toma de decisiones; siendo pocas las entidades que vinculan a la ciudadanía mediante mecanismos y acciones de participación.

Al respecto, desde en el año 2013, la Contraloría General de la República del Perú (CGR) ha incorporado como parte de su compromiso con la MGP el sistema INFObras, alineado al eje transversal del gobierno electrónico, el mismo que busca promover la cercanía del Estado y los ciudadanos, generando transparencia y eficacia durante el desarrollo de obras públicas a nivel nacional. Sin embargo, a la fecha, no se aprecia que las entidades públicas cumplan a cabalidad con el marco normativo de este sistema, lo que a la larga no permite generar el nivel de confianza deseado del ciudadano hacia las entidades; al contrario, se percibe que estas actúan buscando intereses particulares y no el bienestar común de la sociedad.

Para el presente trabajo se consideraron los siguientes objetivos:

Objetivo general:

Evaluar mediante el control social el nivel de cumplimiento normativo del sistema INFObras en la Entidad Prestadora de Servicios de Saneamiento Tacna S.A.

Objetivos específicos:

a) Determinar si las obras en estado situacional "en ejecución" cuentan con su avance físico y financiero actualizado.

b) Determinar si los proyectos de inversión del sistema de ejecución presupuestal del año 2020 se encuentran registrados en el sistema INFObras.

c) Determinar si las obras en estado situacional "finalizada" cuentan con el avance físico al $100 \%$ y con recepción y liquidación de obra. 
Estos objetivos son de gran importancia porque vinculan a la ciudadanía en la aplicación del control social. Se revisa la información almacenada en INFObras, si esta es pertinente y si se encuentra actualizada, generando de esta forma un mecanismo de nivel descentralizado replicable en otras regiones, por los diversos grupos sociales de cada localidad para evidenciar el buen uso y manejo del recurso presupuestal. Del mismo modo, alertando de forma oportuna a las instancias correspondientes si se encuentra algún acto que atente contra la ética y sociedad en su conjunto.

Asimismo, se han considerado los siguientes antecedentes que evidencian la pertenencia y relevancia de la investigación.

A través de su investigación, Pierri y Lafuente (2020) estudiaron la influencia de la implementación de herramientas del gobierno digital dentro de la gestión pública, así como de iniciativas de participación ciudadana en el territorio peruano, midiendo los efectos del programa "Monitores ciudadanos de control" de la CGR y su articulación con los datos de herramientas tecnológicas como la plataforma INFObras y la consulta de inversión del MEF, llegando a la conclusión que existe un impacto positivo y significativo en la reducción de los sobrecostos para obras tercerizadas a firmas constructoras; asimismo, no se encontraron efectos significativos en resultados relativos a la ejecución de las obras.

Por su parte, Gutiérrez (2019), en su estudio para evaluar la calidad en el proceso de gestión durante la ejecución de proyectos de tipo agua potable y saneamiento en la región Huánuco durante el periodo 2011-2015, utilizó dentro de sus fuentes de información el sistema INFObras de la CGR. Llegó a la conclusión que la gestión de la calidad en el desarrollo de proyectos de agua potable y saneamiento básico efectuadas por el gobierno regional de Huánuco, en los periodos antes mencionados fue deficiente. Asimismo, para el estudio, el INFObras brindó información incompleta y tuvo limitaciones en cuanto al registro de data relevante, así como de no contar con dimensiones que puedan brindar información en el seguimiento y monitoreo de obras.

Para Valdez, Melo, Carpio, Sotomayor y Yépez (2017), en su investigación se buscó identificar el nivel de cumplimiento e implementación de la normativa de transparencia y acceso a la información vigente al año de realizado el estudio, realizando un análisis a través de los portales de transparencia estándar en los gobiernos locales en el sur de Puno. Para esto, se consideraron los documentos internos de gestión de la entidad, mecanismo de contrataciones de bienes y servicios, así como el portal INFObras. Se concluyó que las entidades mantenían información incompleta en los portales de transparencia, lo que limitaba el acceso a la información por parte de la ciudadanía. De igual forma, esto generaba desconfianza sobre las acciones que realiza la entidad, las cuales no son transparentadas a la población.

Asimismo, Meléndez (2018) realiza un análisis sobre el grado de incidencia que tiene la participación ciudadana apoyando a las acciones de control gubernamental en la región de Puno. De esto, se aprecia que las veedurías ciudadanas en toda la región sur de ese departamento han generado un ambiental favorable, apoyando la lucha contra la corrupción. La misma que, con el apoyo interinstitucional de las entidades públicas, podría replicarse y generar la vinculación con la ciudadanía, siendo generadora de valor público y de su participación activa.

Respecto a nuestra realidad nacional, Rodríguez (2016), en su investigación "Ausencia de control gubernamental y corrupción de funcionarios públicos, Amantaní", deja claramente establecido que trabajadores de la entidad en calidad de servidores públicos y cargos de confianza, entre el 2011-2014, gozaban de un amplio dominio y decisión respecto a las acciones administrativas desarrolladas en la entidad, por lo que existía un accionar monopólico, evidenciándose la comisión de delitos como: negociación incompatible y aprovechamiento indebido del cargo y colusión. Dentro de los resultados de los exámenes realizados se identificaron además incumplimiento en el adecuado y oportuno registro de información en INFObras sobre las obras que ejecutaba dicha entidad, por lo que se resalta la importancia de este sistema en cuanto al control social que puede ejercer la ciudadanía.

En ese contexto, es preciso resaltar una vez más la importancia que tiene el control social como parte de la participación ciudadana activa en la evaluación de un correcto desarrollo de las obras públicas. Al respecto, el Banco Interamericano de Desarrollo (2004) define a la participación ciudadana como "el conglomerado de procedimientos mediante los cuales la ciudadana realiza una labor fiscalizadora en las actividades concernientes a la toma de decisiones en las entidades nacionales, regionales e inclusive las locales". 
Finalmente, cabe indicar que se ha seleccionado a la Entidad Prestadora de Servicios de Saneamiento Tacna S.A. (EPS Tacna S.A.) por ser la única entidad que brinda el servicio de agua potable y saneamiento en la localidad; además, ejecuta su propio presupuesto en la región Tacna, para disminuir la brecha de saneamiento. Realiza obras que deben de ser desarrolladas dentro de plazos establecidos y contar con la calidad necesaria; sin embargo, esto no se cumple en todos los casos. Por lo tanto, el análisis realizado en esta investigación pretende evidenciar el real cumplimiento normativo del INFObras, considerando que es el portal en el que se almacena la información real de las acciones que se aprecian en el campo de la infraestructura de saneamiento de la región.

\section{MATERIALES Y MÉTODOS}

La investigación es de tipo básica (Hernández, Fernández y Baptista, 2014), con un nivel de investigación descriptivo - explicativo. Busca generar conocimiento evaluando el cumplimiento normativo del registro de obras públicas en el INFObras en la EPS Tacna.

La población considerada en el presente trabajo son 86 obras, las cuales son ejecutadas mediante administración directa y por contrata, registradas en el sistema INFObras, así como 17 proyectos de inversión registrados en el portal del MEF. En relación a las técnicas empleadas para el recojo de información se ha considerado la utilización de un análisis registral, haciendo uso de información disponible en el sistema INFObras y consulta amigable del MEF, concordante con la política de transparencia del Estado peruano. Asimismo, respecto a los materiales y herramientas para el recojo de información, estos se encuentran conformados por la normatividad relacionada al registro de información de obras públicas, así como la normatividad sobre ejecución de obras por administración directa y por contrata, de igual forma por la tabla de calificación (Tabla 1) para el análisis de datos que permitió interpretar los resultados de la investigación.

\section{Tabla 1}

Puntuaciones para calificación del nivel de incumplimiento

\begin{tabular}{ll}
\hline Calificación & Puntuación \\
\hline Alto & $67 \%$ a $100 \%$ \\
Medio & $34 \%$ a $66 \%$ \\
Bajo & $0 \%$ a $33 \%$ \\
\hline
\end{tabular}

Acontinuación, se describen las bases teóricas aplicables en el desarrollo de la investigación:

De la Ley $\mathrm{N}^{\circ}$ 29951, Ley de presupuesto del sector público para el ejercicio fiscal 2013, se desprende lo siguiente:

\section{Disposición complementaria final. \\ Décima octava.}

En esta disposición se menciona que a partir de la fecha de entrada en vigencia de esta ley, todas las entidades públicas tienen el deber de registrar la información de la ejecución de obras en el INFObras, el cual es un sistema de la CGR.

\section{Directiva $\mathrm{N}^{\circ}$ 007-2013-CG/OEA, Registro de información y participación ciudadana en el control de obras públicas - INFObras de 5 de agosto de 2013}

\section{Numeral 1.- Finalidad:}

La finalidad del sistema INFObras es mejorar los mecanismos de control, así como fortalecer la transparencia en lo referente al registro y seguimiento de obras públicas, articulando la publicación de información con las entidades públicas que ejecutan estas, en concordancia incorporar a los ciudadanos como aliados en el control gubernamental verificando el adecuado avance de estas obras en sus localidades. 


\section{Numeral 5.2.- Sistema INFObras:}

Sistema informativo diseñado por la CGR, el cual permite el registro y seguimiento de las obras públicas que cuentan con un expediente técnico aprobado, asimismo, adiciona progresivamente la información de los avances que se desarrollen durante la ejecución de la obra. Se pueden registrar las fechas de inicio y de finalización, los avances físicos y financieros, evidencias fotográficas del desarrollo de la obra, liquidación, operación y mantenimiento, entre otros.

\section{Numeral 5.6.- Deber de las entidades públicas de brindar información:}

Las entidades públicas son las responsables del adecuado y oportuno registro de información relacionada con las obras públicas que estén ejecutando, ya sea por administración directa, contrata u otra modalidad.

\section{Numeral 6.4.- Registro de seguimiento de la obra:}

Las entidades públicas responsables de la ejecución de las obras que se vienen ejecutando o iniciaran su ejecución tienen un plazo para poder realizar el registro de estas, el mismo que vence el último día hábil del mes siguiente en el que se ha efectuado el avance.

\section{Numeral 6.7.- Obligatoriedad del registro:}

Las entidades públicas de forma obligatoria deben de cumplir con el ingreso de información dentro de los plazos previstos por la directiva. En caso no lo realizaran, se inician las acciones sancionadoras correspondientes en referencia al reglamento de infracciones y de sanciones con las que cuenta la CG, teniendo en consideración que es responsabilidad del titular de la entidad el oportuno registro de la información.

De la Directiva $\mathbf{N}^{\circ}$ 011-2015-CG/GPROD, aprobada con Resolución de Contraloría $\mathbf{N}^{\circ}$ 268-2015-CG de 4 de septiembre de 2015. "Servicio de atención de denuncias", se desprenden las siguientes definiciones:

\section{Control Social:}

Consiste en la participación ciudadana de forma activa dentro de los procedimientos en la gestión pública, relacionados al control ciudadano, con la finalidad que puedan participar en los procesos de la entidad, fiscalizando las diversas unidades de la administración pública, detectando situaciones que contravienen las normativas aplicables para cado caso coadyuvando en la lucha contra la corrupción.

De la Resolución de Contraloría N $^{\circ}$ 195-88-CG, aprobado el 18 de julio de 1988, se desprende la obligatoriedad respecto a la recepción y liquidación de obra para considerarla como finalizada.

\section{Artículo 1 $^{\circ}$}

Numeral 11. Esta norma indica que, una vez concluida la obra, se tiene que designar a una comisión que formula el acta de recepción de los trabajos realizados y concluidos, para que después de la suscripción de dicho documento se realice la liquidación técnica y financiera, en un plazo máximo de 30 días luego de haber suscrito el acta.

Del Decreto Supremo $\mathbf{N}^{\circ}$ 344-2018-EF, que aprueba el Reglamento de la Ley $\mathbf{N}^{\circ} 30225$, Ley de contrataciones del Estado del 31 de diciembre de 2018, se desprende la obligatoriedad respecto a la recepción y liquidación de obra para considerarla como finalizada.

\section{Artículo 208 - Recepción de la obra y plazos}

208.5 Previo a la realización de la recepción de obra, en un plazo máximo de 20 días, se tiene que designar a un comité de recepción de obra, el mismo que evalúa el cumplimiento y la pertinencia de lo ejecutado en campo respecto a la infraestructura culminada. 
208.6 Este artículo menciona que una vez culmina la verificación, y en caso no existiesen observaciones, se procede a realizar el acto de recepción de obra.

\section{Artículo $29^{\circ}$ - Liquidación del contrato de obra}

209.9 En este artículo se indica que, para darse la liquidación de obra, no deben existir controversias pendientes de resolución, ya que la existencia de estas no permite realizar la liquidación.

En resumen, los marcos normativos antes citados fueron la base del análisis realizado. Como se ha evidenciado, las entidades públicas tienen la obligación de realizar el registro de información respecto a las obras, su no cumplimiento tiene responsabilidad para el titular de la entidad.

\section{RESULTADOS}

Del total de obras que se encuentran registradas en el INFObras en la EPS Tacna, así como los proyectos registrados en el sistema de ejecución presupuestal para el año 2020, se ha realizado el proceso de verificación de cumplimiento normativo al amparo del control social. Respecto al mismo, los resultados se muestran a continuación:

\section{Obras en estado situacional "en ejecución”, cuentan con avance (físico y financiero actualizado).}

De la revisión de información de las obras públicas registradas en el INFObras, las mismas que son de acceso público al amparo del numeral 6.8, que define la inserción de la participación de la ciudadanía en la ejecución de obras públicas de la Directiva que habilita el control social de parte de los ciudadanos de forma activa en las actividades que ejecuta la gestión, así como el control público, se ha determinado que:

37 obras públicas tienen un estado situacional “en ejecución”. Sobre esto, según la directiva, se hace mención que la información deberá ser actualizada con plazo máximo al último día hábil del mes que continúa al último avance realizado, lo cual no ha sido cumplido por la entidad evaluada.

A continuación, en la tabla 2, se aprecia el estado situacional de las 37 obras que se encuentran en ejecución a enero de 2021, realizando el análisis del cumplimiento normativo del numeral 6.4.4 de la directiva.

\section{Tabla 2}

Obras con estado situacional "en ejecución” que incumplen el numeral 6.4.4. de la directiva

\begin{tabular}{|c|c|c|c|c|c|c|c|c|}
\hline $\mathbf{N}^{\circ}$ & $\begin{array}{c}\text { Código } \\
\text { unificado }\end{array}$ & $\begin{array}{l}\text { Código } \\
\text { INFObras }\end{array}$ & $\begin{array}{c}\text { Monto de la } \\
\text { inversión (S/) }\end{array}$ & $\begin{array}{c}\text { Último } \\
\text { registro } \\
\text { avance } \\
\text { físico real }\end{array}$ & $\begin{array}{c}\text { Último } \\
\text { avance } \\
\text { físico real } \\
\text { acumulado }\end{array}$ & $\begin{array}{l}\text { Registra el } \\
\text { Acta de } \\
\text { recepción de } \\
\text { obra }\end{array}$ & $\begin{array}{c}\text { Registra el } \\
\text { Documento } \\
\text { de } \\
\text { liquidación } \\
\text { de obra }\end{array}$ & $\begin{array}{c}\text { Numeral } \\
\text { 6.4.4. de } \\
\text { la } \\
\text { Directiva }\end{array}$ \\
\hline 1 & 2146653 & 5790 & 71374.75 & abr-12 & $63.80 \%$ & No & No & $\mathrm{N}^{* *}$ \\
\hline 2 & 2146654 & 5791 & 270702.70 & mar-12 & $69.84 \%$ & No & No & $\mathrm{N}^{* *}$ \\
\hline 3 & 2161473 & 7705 & 417919.69 & abr-13 & $78.68 \%$ & No & No & $\mathrm{N}^{* *}$ \\
\hline 4 & 2154335 & 8295 & 1328477.54 & ene-13 & $79.30 \%$ & No & No & $\mathrm{N}^{* *}$ \\
\hline 5 & 2217003 & 14297 & 223335.12 & jun-13 & $78.83 \%$ & No & No & $\mathrm{N} * *$ \\
\hline 6 & 2108995 & 16332 & 17768337.98 & sep-13 & $72.76 \%$ & No & No & $\mathrm{N}^{* *}$ \\
\hline 7 & 2148018 & 18349 & 536195.65 & jul-14 & $48.81 \%$ & No & No & $\mathrm{N}^{* *}$ \\
\hline 8 & 2146647 & 20950 & 764672.00 & $\mathrm{~S}-\mathrm{R}^{*}$ & $\mathrm{~S}-\mathrm{R} *$ & No & No & $\mathrm{N} * *$ \\
\hline 9 & 2117041 & 28527 & 945512.26 & nov-15 & $59.15 \%$ & No & No & $\mathrm{N} * *$ \\
\hline 10 & 2161474 & 58186 & 3157661.61 & abr-17 & $78.85 \%$ & No & No & $\mathrm{N}^{* *}$ \\
\hline 11 & 2201405 & 60847 & 968722.61 & dic-16 & $73.19 \%$ & No & No & $\mathrm{N} * *$ \\
\hline 12 & 2167693 & 62027 & 5191889.37 & feb-17 & $51.80 \%$ & No & No & $\mathrm{N}^{* *}$ \\
\hline 13 & - & 102935 & 929803.60 & $\mathrm{~S}-\mathrm{R}^{*}$ & S-R* & No & No & $\mathrm{N} * *$ \\
\hline 14 & 2466053 & 119899 & 6001254.04 & sep-20 & $24.03 \%$ & No & No & $\mathrm{N}^{* *}$ \\
\hline 15 & 2093199 & 5783 & 4176558.16 & may-12 & $98.74 \%$ & No & No & $\mathrm{N}^{* *}$ \\
\hline 16 & 2094833 & 5784 & 3808550.50 & feb-12 & $88.75 \%$ & No & No & $\mathrm{N}^{* *}$ \\
\hline 17 & 2149928 & 5785 & 965625.66 & mar-12 & $99.79 \%$ & No & No & $\mathrm{N}^{* *}$ \\
\hline 18 & 2153940 & 5787 & 1579644.00 & may-12 & $5.87 \%$ & No & No & $\mathrm{N} * *$ \\
\hline
\end{tabular}




\begin{tabular}{|c|c|c|c|c|c|c|c|c|}
\hline $\mathbf{N}^{\circ}$ & $\begin{array}{c}\text { Código } \\
\text { unificado }\end{array}$ & $\begin{array}{l}\text { Código } \\
\text { INFObras }\end{array}$ & $\begin{array}{c}\text { Monto de la } \\
\text { inversión (S/) }\end{array}$ & $\begin{array}{l}\text { Último } \\
\text { registro } \\
\text { avance } \\
\text { fisico real }\end{array}$ & $\begin{array}{c}\text { Último } \\
\text { avance } \\
\text { físico real } \\
\text { acumulado }\end{array}$ & $\begin{array}{l}\text { Registra el } \\
\text { Acta de } \\
\text { recepción de } \\
\text { obra }\end{array}$ & $\begin{array}{c}\text { Registra el } \\
\text { Documento } \\
\text { de } \\
\text { liquidación } \\
\text { de obra }\end{array}$ & $\begin{array}{c}\text { Numeral } \\
\text { 6.4.4. de } \\
\text { la } \\
\text { Directiva }\end{array}$ \\
\hline 19 & 2153929 & 5789 & 589368.91 & jun-12 & $98.81 \%$ & No & No & $\mathrm{N}^{* *}$ \\
\hline 20 & 2148046 & 5792 & 136144.53 & jul-12 & $88.33 \%$ & No & No & $\mathrm{N}^{* *}$ \\
\hline 21 & 2117064 & 6322 & 1000000.00 & ago-12 & $98.72 \%$ & No & No & $\mathrm{N}^{* *}$ \\
\hline 22 & 2162451 & 8299 & 283713.64 & jul-13 & $98.54 \%$ & No & No & $\mathrm{N}^{* *}$ \\
\hline 23 & 2146161 & 10270 & 896219.42 & jun-13 & $97.32 \%$ & No & No & $\mathrm{N}^{* *}$ \\
\hline 24 & 2162452 & 15112 & 231856.81 & jul-13 & $78.33 \%$ & No & No & $\mathrm{N}^{* *}$ \\
\hline 25 & 2167712 & 15230 & 1436540.03 & oct-13 & $94.82 \%$ & No & No & $\mathrm{N}^{* *}$ \\
\hline 26 & 2217013 & 15384 & 898852.22 & nov-14 & $95.78 \%$ & No & No & $\mathrm{N}^{* *}$ \\
\hline 27 & 2217011 & 15621 & 418271.75 & feb-14 & $99.68 \%$ & No & No & $\mathrm{N}^{* *}$ \\
\hline 28 & 2159524 & 15818 & 1001466.02 & ene-14 & $90.38 \%$ & No & No & $\mathrm{N}^{* *}$ \\
\hline 29 & 2148022 & 18457 & 156504.64 & feb-14 & $92.21 \%$ & No & No & $\mathrm{N}^{* *}$ \\
\hline 30 & 2162453 & 18580 & 1009580.27 & dic-13 & $99.89 \%$ & No & No & $\mathrm{N}^{* *}$ \\
\hline 31 & 2167691 & 21147 & 830677.23 & jun-14 & $87.37 \%$ & No & No & $\mathrm{N}^{* *}$ \\
\hline 32 & 2162453 & 26145 & 420392.00 & oct-14 & $90.51 \%$ & No & No & $\mathrm{N}^{* *}$ \\
\hline 33 & 2134611 & 32750 & 186385.58 & dic-11 & $99.69 \%$ & No & No & $\mathrm{N}^{* *}$ \\
\hline 34 & 2094746 & 32751 & 490593.78 & may-11 & $83.83 \%$ & No & No & $\mathrm{N}^{* *}$ \\
\hline 35 & 2154335 & 33646 & 10513838.18 & nov-15 & $78.84 \%$ & No & No & $\mathrm{N}^{* *}$ \\
\hline 36 & 2447440 & 32733 & 2154134.47 & nov-20 & $68.50 \%$ & No & No & $\mathrm{N}^{* *}$ \\
\hline 37 & 2370531 & 64984 & 2385965.69 & nov-20 & $67.13 \%$ & No & No & $\mathrm{N}^{* *}$ \\
\hline
\end{tabular}

Nota: $\mathrm{N}^{* *}$ que significaba NO CUMPLE / S-R* que significaba SIN REGISTRO

\section{Proyectos de inversión del sistema de ejecución presupuestal del año 2020 se encuentran registrados en el sistema INFObras}

De la revisión de información de proyectos de inversión registrados en el sistema de ejecución presupuestal al año 2020, se ha evidenciado que aquellos que cuentan con expediente técnico aprobados fueron registrados en el sistema INFObras. Únicamente, no están registrados los proyectos que se encuentran en etapa de elaboración de expediente técnico, todo esto concordante con lo indicado en la directiva aplicable para obras públicas, indistintamente de la modalidad de contratación.

Obras en estado situacional "finalizado" cuentan con el avance físico al $100 \%$ y con recepción y liquidación de obras

De la revisión de información de obras públicas registradas en el sistema INFObras, las mismas que son de acceso público para la aplicación del control social que realiza la ciudadanía de forma activa en los procesos de gestión y control público concordante con el numeral 6.8 que ampara el derecho de participación ciudadana controlando la ejecución de las acciones que se realizan en las obras públicas de la directiva, se ha determinado que:

22 obras públicas tienen un estado situacional "finalizado", presentando una ejecución de gasto menor al $100 \%$. Asimismo, no cuentan con documentos registrados sobre la recepción ni liquidación de la obra. Lo que haría suponer que no se habrían finalizado o, si finalizaron, habría sido de forma irregular según lo indicado en el numeral 6.4.1 de la directiva.

A continuación, en la tabla 3 , se aprecia el estado situacional de las 22 obras que se encuentran finalizadas con un gasto financiero menor al $100 \%$ a enero de 2021 que no cuentan con sustento de recepción ni liquidación de obra, en concordancia a la verificación del cumplimiento normativo del numeral 6.4.1 de la directiva. 
Tabla 3

Obras con estado situacional "finalizado" con avance físico real menor al 100\% que incumplen el numeral 6.4.1. de la directiva

\begin{tabular}{|c|c|c|c|c|c|c|c|c|}
\hline $\mathbf{N}^{\circ}$ & $\begin{array}{c}\text { Código } \\
\text { unificado }\end{array}$ & $\begin{array}{l}\text { Código } \\
\text { INFObras }\end{array}$ & $\begin{array}{c}\text { Monto de la } \\
\text { inversión (S/) }\end{array}$ & $\begin{array}{l}\text { Último } \\
\text { registro } \\
\text { avance } \\
\text { físico } \\
\quad \text { real } \\
\end{array}$ & $\begin{array}{c}\text { Último } \\
\text { avance físico } \\
\text { real } \\
\text { acumulado }\end{array}$ & $\begin{array}{l}\text { Registra el } \\
\text { Acta de } \\
\text { recepción } \\
\text { de obra }\end{array}$ & $\begin{array}{c}\text { Registra el } \\
\text { Documento } \\
\text { de } \\
\text { liquidación } \\
\text { de obra } \\
\end{array}$ & $\begin{array}{c}\text { Numeral } \\
6.4 .1 . \text { de la } \\
\text { Directiva }\end{array}$ \\
\hline 1 & 2235790 & 64984 & 5375599,00 & ene-18 & $33.51 \%$ & No & No & $\mathrm{N} * *$ \\
\hline 2 & 2201410 & 69758 & 3766100,37 & ago-18 & $68.09 \%$ & No & No & $\mathrm{N}^{* *}$ \\
\hline 3 & 2153932 & 28425 & 1745798,74 & nov-15 & $98.82 \%$ & No & No & $\mathrm{N} * *$ \\
\hline 4 & 2148040 & 31783 & 830212,14 & sep-15 & $96.53 \%$ & No & No & $\mathrm{N}^{* *}$ \\
\hline 5 & 2155516 & 33353 & 1028862,94 & jul-16 & $94.12 \%$ & No & No & $\mathrm{N} * *$ \\
\hline 6 & 2162453 & 42052 & S-R* & sep-15 & $99.78 \%$ & No & No & $\mathrm{N}^{* *}$ \\
\hline 7 & 2117043 & 48118 & S-R* & sep-16 & $94.73 \%$ & No & No & $\mathrm{N}^{* *}$ \\
\hline 8 & 2162452 & 48137 & S-R* & oct-16 & $90.88 \%$ & No & No & $\mathrm{N}^{* *}$ \\
\hline 9 & 2294790 & 50116 & $\mathrm{~S}-\mathrm{R}^{*}$ & ene-16 & $99.63 \%$ & No & No & $\mathrm{N} * *$ \\
\hline 10 & 2217018 & 58185 & 2725789,00 & abr-17 & $98.77 \%$ & No & No & $\mathrm{N}^{* *}$ \\
\hline 11 & 2167694 & 58658 & S-R* & ene-17 & $94.03 \%$ & No & No & $\mathrm{N}^{* *}$ \\
\hline 12 & 2323828 & 71222 & 3020900,00 & ago-18 & $93.66 \%$ & No & No & $\mathrm{N}^{* *}$ \\
\hline 13 & 2201415 & 76966 & S-R* & dic-17 & $96.20 \%$ & No & No & $\mathrm{N}^{* *}$ \\
\hline 14 & 2117043 & 77094 & S-R* & sep-17 & $99.13 \%$ & No & No & $\mathrm{N}^{* *}$ \\
\hline 15 & 2335864 & 81375 & $S-R^{*}$ & mar-18 & $95.65 \%$ & No & No & $\mathrm{N}^{* *}$ \\
\hline 16 & 2117043 & 81380 & $S-R^{*}$ & dic-18 & $99.53 \%$ & No & No & $\mathrm{N}^{* *}$ \\
\hline 17 & 2400735 & 85203 & S-R* & jun-18 & $99.66 \%$ & No & No & $\mathrm{N}^{* *}$ \\
\hline 18 & 2167693 & 87020 & $\mathrm{~S}-\mathrm{R}^{*}$ & mar-19 & $99.99 \%$ & No & No & $\mathrm{N}^{* *}$ \\
\hline 19 & 2370591 & 101805 & S-R* & jun-19 & $97.47 \%$ & No & No & $\mathrm{N} * *$ \\
\hline 20 & 2370518 & 104339 & $S-R^{*}$ & sep-19 & $99.97 \%$ & No & No & $\mathrm{N}^{* *}$ \\
\hline 21 & 2370379 & 115170 & S-R* & jul-20 & $96.17 \%$ & No & No & $\mathrm{N}^{* *}$ \\
\hline 22 & 2410978 & 121809 & S-R* & sep-20 & $97.97 \%$ & No & No & $\mathrm{N} * *$ \\
\hline
\end{tabular}

Nota: $N^{* *}$ que significaba NO CUMPLE / S-R* que significaba SIN REGISTRO

24 obras públicas tienen un estado situacional "finalizado", presentando una ejecución de gasto al $100 \%$; sin embargo, no cuentan con documento registrado sobre la recepción ni liquidación de obra. Esto denotaría que no habrían finalizado o, si finalizaron, habría sido de forma irregular según lo indicado en el numeral 6.4.1 de la directiva.

En la tabla 4, se aprecia el estado situacional de las 24 obras que se encuentran finalizadas con un gasto financiero de $100 \%$ a enero de 2021. Sin embargo, no cuentan con sustento de recepción ni liquidación de obra, en concordancia a la verificación del cumplimiento normativo del numeral 6.4.1 de la directiva. 
Tabla 4

Obras con estado situacional "finalizado" con avance físico real al 100\% que incumplen el numeral 6.4.1. de la directiva

\begin{tabular}{|c|c|c|c|c|c|c|c|c|}
\hline $\mathbf{N}^{\circ}$ & $\begin{array}{c}\text { Código } \\
\text { unificado }\end{array}$ & $\begin{array}{l}\text { Código } \\
\text { INFObras }\end{array}$ & $\begin{array}{c}\text { Monto de la } \\
\text { inversión (S/) }\end{array}$ & $\begin{array}{c}\text { Último } \\
\text { registro } \\
\text { avance } \\
\text { físico real }\end{array}$ & $\begin{array}{c}\text { Último } \\
\text { avance } \\
\text { físico real } \\
\text { acumulado }\end{array}$ & $\begin{array}{l}\text { Registra el } \\
\text { Acta de } \\
\text { recepción } \\
\text { de obra }\end{array}$ & $\begin{array}{c}\text { Registra el } \\
\text { Documento } \\
\text { de } \\
\text { liquidación } \\
\text { de obra }\end{array}$ & $\begin{array}{c}\text { Numeral } \\
\text { 6.4.1. de la } \\
\text { Directiva }\end{array}$ \\
\hline 1 & 2146649 & 5778 & 537569.16 & dic-11 & $100 \%$ & No & No & $\mathrm{N} * *$ \\
\hline 2 & 2146651 & 5779 & 493097.38 & dic-11 & $100 \%$ & No & No & $\mathrm{N}^{* *}$ \\
\hline 3 & 2146652 & 5780 & 617828.32 & dic-11 & $100 \%$ & No & No & $\mathrm{N}^{* *}$ \\
\hline 4 & 2117064 & 5781 & 793535.00 & ene-12 & $100 \%$ & No & No & $\mathrm{N}^{* *}$ \\
\hline 5 & 2089706 & 5782 & 469819.54 & ene-12 & $100 \%$ & No & No & $\mathrm{N}^{* *}$ \\
\hline 6 & 2094749 & 5786 & 2778210.93 & feb-13 & $100 \%$ & No & No & $\mathrm{N}^{* *}$ \\
\hline 7 & 2153928 & 5788 & 1720837.13 & ene-13 & $100 \%$ & No & No & $\mathrm{N}^{* *}$ \\
\hline 8 & 2148035 & 15546 & 879562.41 & sep-13 & $100 \%$ & No & No & $\mathrm{N}^{* *}$ \\
\hline 9 & 2158008 & 28415 & 1556378.80 & nov-15 & $100 \%$ & No & No & $\mathrm{N}^{* *}$ \\
\hline 10 & 2117064 & 28416 & 5014393.76 & dic-15 & $100 \%$ & No & No & $\mathrm{N}^{* *}$ \\
\hline 11 & 2167694 & 30271 & 2420271.50 & oct- 15 & $100 \%$ & No & No & $\mathrm{N}^{* *}$ \\
\hline 12 & 2162452 & 32666 & 2368295.93 & oct-15 & $100 \%$ & No & No & $\mathrm{N}^{* *}$ \\
\hline 13 & 2146650 & 32752 & 44222.41 & ene-12 & $100 \%$ & No & No & $\mathrm{N} * *$ \\
\hline 14 & 2262533 & 41922 & 298944.36 & nov-15 & $100 \%$ & No & No & $\mathrm{N}^{* *}$ \\
\hline 15 & 2217017 & 52077 & 2329100.26 & oct-16 & $100 \%$ & No & No & $\mathrm{N} * *$ \\
\hline 16 & 2153930 & 58643 & 3664905.95 & jul-17 & $100 \%$ & No & No & $\mathrm{N}^{* *}$ \\
\hline 17 & 2201415 & 62432 & 984132.97 & mar-17 & $100 \%$ & No & No & $\mathrm{N}^{* *}$ \\
\hline 18 & 2296089 & 75292 & 905278.16 & ene-18 & $100 \%$ & No & No & $\mathrm{N} * *$ \\
\hline 19 & 2322065 & 75309 & 884140.32 & feb-18 & $100 \%$ & No & No & $\mathrm{N}^{* *}$ \\
\hline 20 & 2252101 & 79903 & 325862.65 & ene-18 & $100 \%$ & No & No & $\mathrm{N}^{* *}$ \\
\hline 21 & 2370556 & 93922 & 158645.86 & ene-19 & $100 \%$ & No & No & $\mathrm{N}^{* *}$ \\
\hline 22 & 2167693 & 102986 & 5191889.37 & jun-19 & $100 \%$ & No & No & $\mathrm{N}^{* *}$ \\
\hline 23 & 2201406 & 107514 & 524803.48 & oct-19 & $100 \%$ & No & No & $\mathrm{N}^{* *}$ \\
\hline 24 & 2410862 & 119326 & 95218.55 & dic-19 & $100 \%$ & No & No & $\mathrm{N}^{* *}$ \\
\hline
\end{tabular}

Nota: N** que significaba NO CUMPLE / S-R* que significaba SIN REGISTRO

\section{DISCUSIÓN}

Para el presente trabajo de investigación se analizaron 86 obras públicas que se encuentran en el sistema INFObras, correspondientes a la EPS Tacna, de las cuales el $96.5 \%$ presenta información incompleta en la actualización de datos concordante con la directiva. Por tanto, según la tabla 1, tiene un alto nivel de incumplimiento normativo, lo antes indicado se encuentra sintetizados en la tabla 5.

Dentro de las 86 obras registradas, según la información de INFObras, se encuentra que fueron desarrolladas por administración directiva y por contrata. 
Tabla 5

Resumen de obras que presentan incumplimiento normativo respecto a la directiva

\begin{tabular}{|c|c|c|c|c|c|c|}
\hline $\begin{array}{c}\text { Registro } \\
\text { INFObras }\end{array}$ & Cantidad & Porcentaje & $\begin{array}{c}\text { Incumplimiento } \\
\text { normativo a la } \\
\text { directiva } \\
\end{array}$ & Cantidad & Porcentaje & Comentario \\
\hline $\begin{array}{l}\text { Obras en } \\
\text { ejecución }\end{array}$ & $37(100 \%)$ & $43.0 \%$ & $\begin{array}{c}\text { Obras en } \\
\text { ejecución con } \\
\text { incumplimiento }\end{array}$ & $37(100 \%)$ & $43.0 \%$ & $\begin{array}{l}\text { Incumplimiento del } \\
\text { Numeral 6.4.4. de la } \\
\text { directiva }\end{array}$ \\
\hline $\begin{array}{l}\text { Obras sin } \\
\text { ejecución }\end{array}$ & $3(100 \%)$ & $3.5 \%$ & $\begin{array}{l}\text { Obras sin } \\
\text { ejecución con } \\
\text { incumplimiento }\end{array}$ & $0(0 \%)$ & $0.0 \%$ & \\
\hline $\begin{array}{l}\text { Obras } \\
\text { finalizadas }\end{array}$ & $46(100 \%)$ & $53.5 \%$ & $\begin{array}{l}\text { Obras finalizadas } \\
\text { con } \\
\text { incumplimiento }\end{array}$ & $46(100 \%)$ & $53.5 \%$ & $\begin{array}{l}\text { Incumplimiento del } \\
\text { Numeral 6.4.1. de la } \\
\text { directiva }\end{array}$ \\
\hline $\begin{array}{c}\text { Total, de Obras } \\
\text { registradas }\end{array}$ & 86 & $100 \%$ & $\begin{array}{l}\text { Total de obras } \\
\text { con } \\
\text { incumplimiento }\end{array}$ & 83 & $96.5 \%$ & $\begin{array}{l}\text { ALTO NIVEL DE } \\
\text { INCUMPLIMIENTO } \\
\text { (Tabla 1) }\end{array}$ \\
\hline
\end{tabular}

En tal sentido, de las 37 obras públicas que se encuentran en estado situacional "en ejecución", que representan un $43 \%$ del total de obras registradas en el sistema INFObras, el $100 \%$ (alto nivel de incumplimiento) presenta desactualización de información respecto al numeral 6.4.4. de la directiva; la cual dentro de su contenido menciona que "las entidades tienen un plazo máximo para realizar el registro de obras nuevas y de avances hasta el último día hábil del siguiente mes de haberse realizado los avances". De la evaluación realizada, se han identificado obras que tienen como última fecha de registro entre febrero de 2012, 2013, 2014, 2015, 2016, 2017 a noviembre de 2020. Por tanto, habiéndose realizado el control social al sistema INFObras en el mes de enero 2021, la actualización debió darse en diciembre 2020. Tal situación limita el acceso a la ciudadanía a su derecho de participación ciudadana mediante el control social de obras públicas.

Asimismo, de las 46 obras públicas que se encuentran en el estado situacional de "finalizada", las cuales representan un $53.5 \%$ del total de obras registradas en el sistema INFObras, el $100 \%$ (alto nivel de incumplimiento) presentan desactualización de información respecto al numeral 6.4.1. de la directiva; considerando que dentro de los alcances de este numeral se establece que deben ser registrados los datos de la obra correspondiente a la ejecución de gasto, recepción de obra y liquidación. Del mismo modo, en concordancia con la Resolución de Contraloría $\mathrm{N}^{\circ}$ 195-88-CG y el Reglamento de la Ley $\mathrm{N}^{\circ} 30225$, ley de contrataciones del Estado, correspondiente a la recepción y liquidación de obra, la actualización de datos debe darse culminada satisfactoriamente la etapa de ejecución de la obra. Lo que denotaría que las 46 obras no habrían culminado de forma satisfactoria o que la entidad habría omitido el cumplimiento de dichas normativas, incurriendo en una omisión de funciones, limitando el derecho de participación ciudadana mediante el control social de obras públicas.

En base a lo señalado, se puede concluir que la EPS Tacna tiene un alto nivel de incumplimiento, debido a que no realiza el adecuado registro, seguimiento y actualización de la información de las obras públicas que viene ejecutando desde la implementación de la normativa. Asimismo, no cumple con la finalidad de la mencionada directiva, respecto al fortalecimiento del control y la transparencia, haciendo que el ciudadano se convierta en un aliado estratégico dentro de las acciones de control gubernamental en obras públicas.

\section{CONCLUSIONES}

La aplicación del control social en la evaluación de la información del sistema INFObras de la CGR y del sistema de ejecución presupuestal del MEF permitió evidencia un alto nivel de incumplimiento normativo en el registro de obras públicas del sistema INFObras, por parte de la EPS Tacna, limitando la ejecución del derecho de participación ciudadana a la población de la región Tacna, es decir, conocer si las obras en su región se estén desarrollando dentro de los plazos establecidos, con la calidad requerida y cumpliendo su finalidad pública. 
Se ha determinado que 37 obras públicas se encuentran en estado situacional "en ejecución”, de las cuales el 100 $\%$ cuenta con registro de avance físico y financiero desactualizado, teniendo un alto nivel de incumplimiento con relación al numeral 6.4.4. de la directiva. Considerando que la información debe estar actualizada con fecha máxima al último día hábil del mes siguiente de culminada la obra, se encontró que al momento de realizar el control social en el sistema INFObras figuraban como últimas fechas de registro desde febrero de 2012 a noviembre de 2020, debiendo ser actualizada la información en diciembre de 2020.

No se han identificado proyectos de inversión en el sistema de ejecución presupuestal del MEF que no se encuentren registrados en el Sistema INFObras. Por tanto, no habría incumplimiento normativo en el adecuado registro de la información, concordante con lo indicado en la décimo octava disposición complementaria final de la Ley $\mathrm{N}^{\circ} 29951$.

Se han identificado que 22 obras públicas se encuentran en estado situacional "finalizada", las cuales cuentan con un avance físico acumulado menor a $100 \%$. De estas, el 100\% no cuenta con el registro de algún documento que acredite la recepción ni la liquidación de obra, lo que indica un alto nivel de incumplimiento con relación al numeral 6.4.1 de la directiva. Considerando que para culminar con el proceso de ejecución de obra, esta debe ser recepcionada y posteriormente liquidada; en ese sentido, no es posible determinar si las 22 obras identificadas vienen cumpliendo satisfactoriamente su finalidad pública. Asimismo, se desconoce el motivo por el cual no se encuentran al $100 \%$ de su avance físico.

Se ha determinado que 24 obras públicas se encuentran en estado situacional "finalizado", las cuales cuentan con un avance físico acumulado de $100 \%$. Sin embargo, no registran documento alguno que acredite que fueron recepcionadas ni liquidadas, por lo presentan un alto nivel de incumplimiento normativo del numeral 6.4.1 de la directiva. Al respecto, según indican las normas vigentes para obras por administración directica y por contrata, para considerar que un proyecto se encuentre finalizado debe de haberse realizado satisfactoriamente el proceso de recepción de obra y posterior liquidación. Por tanto, se aprecia que estas obras no habrían culminado de forma satisfactoria su ejecución, no cumpliendo de esta forma su finalidad pública o en su defecto que la EPS Tacna esté incumpliendo en transparentar la información, limitando los derechos de participación social de los ciudadanos en la región Tacna.

\section{REFERENCIAS}

Banco Interamericano de Desarrollo (2004). Estrategias para promover la participación ciudadana en las actividades del Banco. https://dhls.hegoa.ehu.eus/documents/4608

Contraloría General de la República (18 de julio de 1988). Resolución de Contraloría № 195-88-CG, ejecución de obras públicas por administración directa.

Contraloría General de la República (5 de agosto de 2013). Directiva N 007-2013-CG/OEA, registro de información y participación ciudadana en el control de obras públicas - INFObras.

Contraloría General de la República (4 de setiembre de 2015). Directiva Nº 011-2015-CG/GPROD “Servicio de atención de denuncias".

Gutiérrez, B. (2019). Indicadores de calidad para la gestión de ejecución de proyectos de saneamiento básico, Huánuco, 2011 - 2015 . Revista Gobierno y Gestión Pública, 6 (1), $125-159$. https://doi.org/10.24265/iggp.2019.v6n1.06

Hernández, R., Fernández, C. y Baptista, M. (2014). Metodología de la investigación. McGraw-Hill Educación.

Meléndez, A. (2018). Nivel de aplicación de la norma sobre veeduría ciudadana para el control gubernamental en los gobiernos locales provinciales de la 123 zona sur de la región Puno - 2016 [Tesis de maestría, Universidad Nacional del Altiplano, Puno, Perú].

Ministerio de Economía y Finanzas (2012). Ley $\mathrm{N}^{\circ}$ 29951, ley del presupuesto del sector público para el ejercicio fiscal 2013.

Ministerio de Economía y Finanzas (31 de diciembre de 2018). Decreto Supremo N 344-2018-EF, que aprueba el reglamento de la Ley $\mathrm{N}^{\circ} 30225$, ley de contrataciones del estado. 
Organización de las Naciones Unidas (2015). Proyecto de documento final de la cumbre de las Naciones Unidas para la aprobación de la agenda para el desarrollo después de 2015. http://www.un.org/es/comun/docs/?symbol=A/69/L.85

Pierri, G. y Lafuente, M. (2020). El impacto de Infobras y el control ciudadano en la eficiencia de la ejecución de obras públicas en Perú. Gobierno digital y corrupción. https://publications.iadb.org/publications/spanish/document/Gobierno-digital-y-corrupcion-Elimpacto-de-InfObras-y-el-control-ciudadano-en-la-eficiencia-de-la-ejecucion-de-obras-publicas-enPeru.pdf

Rodríguez, J. (2016). Ausencia de control gubernamental y corrupción de funcionarios públicos, Amantaní. Revista Científica Investigación Andina, 16(2), 123-135.

Valdez, K., Melo, M., Carpio, J., Sotomayor, J., y Yepez, K. (2017). Evaluación de la implementación de los portales de transparencia estándar en concordancia con la normativa en las municipalidades provinciales del sur de Puno. Revista de Investigación, 6(4).

http://revistaepgunapuno.org/index.php/investigaciones/article/view/141 